\title{
MASSIVE HAEMORRHAGE DUE TO SPONTANEOUS RUPTURE OF A GIANT RENAL ANGIOMYOLIPOMA - REPORT OF A RARE CASE AND REVIEW OF THE LITERATURE
}

\author{
Iv. Novakov* \\ Department of Special Surgey, Medical University - Plovdiv, Bulgaria
}

\begin{abstract}
Massive hemorrhage due to spontaneous rupture of a renal angiomyolipoma is a rare, but the most severe complication of this unusual tumor.

The aim of this publication is to present a rare case of massive retroperitoneal hemorrhage and hemoperitoneum due to spontaneous rupture of giant renal angiomyolipoma.

Case presentation: A 20-year-old woman, with a previous diagnosis of tuberous sclerosis and sudden onset of severe abdominal pain is presented. Median laparotomy on emergency was performed, with operative finding of hemoperitoneum and a giant ruptured retroperitoneal tumor with retroperitoneal hematoma. Gross pathology and histological examination determined the origin of the retroperitoneal tumor mass - angiomyolipoma, complicated with rupture and severe bleeding.

In conclusion, this case presents rare, but the most severe complication of renal angiomyolipomas spontaneous rupture of the tumor with life-threatening retroperitoneal bleeding.
\end{abstract}

Key words: kidney tumor, tuberous sclerosis complex, retroperitoneal hemorrhage, Wunderlich's syndrome.

\section{INTRODUCTION}

Angiomyolipomas of kidneys are benign renal tumours, composed of mature adipose tissue, smooth muscle and abnormally thick-walled blood vessels. For this reason they are also known as renal hamartomas (1-3). Although most angiomyolipomas are asymptomatic, symptoms develop in patients when tumor size reaches $4 \mathrm{~cm}$ or greater. Complications from angiomyolipomas are rare but often severe, depending on the size and content of the angiomyolipoma. They are associated with bleeding, compression and necrosis. The most severe complication is massive retroperitoneal hemorrhage due to spontaneous rupture of this unusual tumor (4-6).

\section{OBJECTIVE}

The aim is this publication is to present a rare case of massive retroperitoneal hemorrhage

Ivan P. Novakov, Department of Special Surgey, Medical University - Plovdiv, Bulgaria, E-mail: inovakov2003@yahoo.com, mobile phone: $+359887575487$ and hemoperitoneum due to spontaneous rupture of giant renal angiomyolipoma associated with tuberous sclerosis complex. On the base of the presented case, the review of the literature onto this problem is made.

\section{CASE PRESENTATION}

A 20-year-old woman, with a previous diagnosis of tuberous sclerosis, was presented to the emergency department of the University Hospital "St. George"- Plovdiv with sudden onset of severe abdominal pain. There was no history of trauma.

On physical examination the woman appeared acutely ill with a blood pressure of $90 / 60 \mathrm{~mm}$. $\mathrm{Hg}$ and a pulse of 120 per minute. The abdomen was soft with markedly diminished peristaltic sounds, severe tenderness in the right flank and no definite palpable mass. Facial hypomelanotic macules and angiofibromas were established.

Blood analysis showed hemoglobin $84.2 \mathrm{~g} / \mathrm{l}$, hematocrit $0.24 \quad 1 / 1$, white blood cells 
$26.8 \times 10^{9} / 1$ and creatinin level of $120 \mu \mathrm{mol} / 1$.

Abdominal sonography appeared to suggest intraperitoneal haemorrhage. Heterogeneous solid mass, with maximum diameter of $18 \mathrm{~cm}$ in the right abdominal area, with probable origin from the right kidney was observed.

Unenhanced abdominal computerized axial tomography showed bilateral abnormally enlarged kidneys (maximum diameter of $23 \mathrm{~cm}$ for the right and $17 \mathrm{~cm}$ for the left). Both kidneys were presented as tumor masses with cystic transformation (with density of fat, muscles, and vessels). A giant right-sided retroperitoneal hematoma and intraperitoneal fluid collection were established (Figure 1).

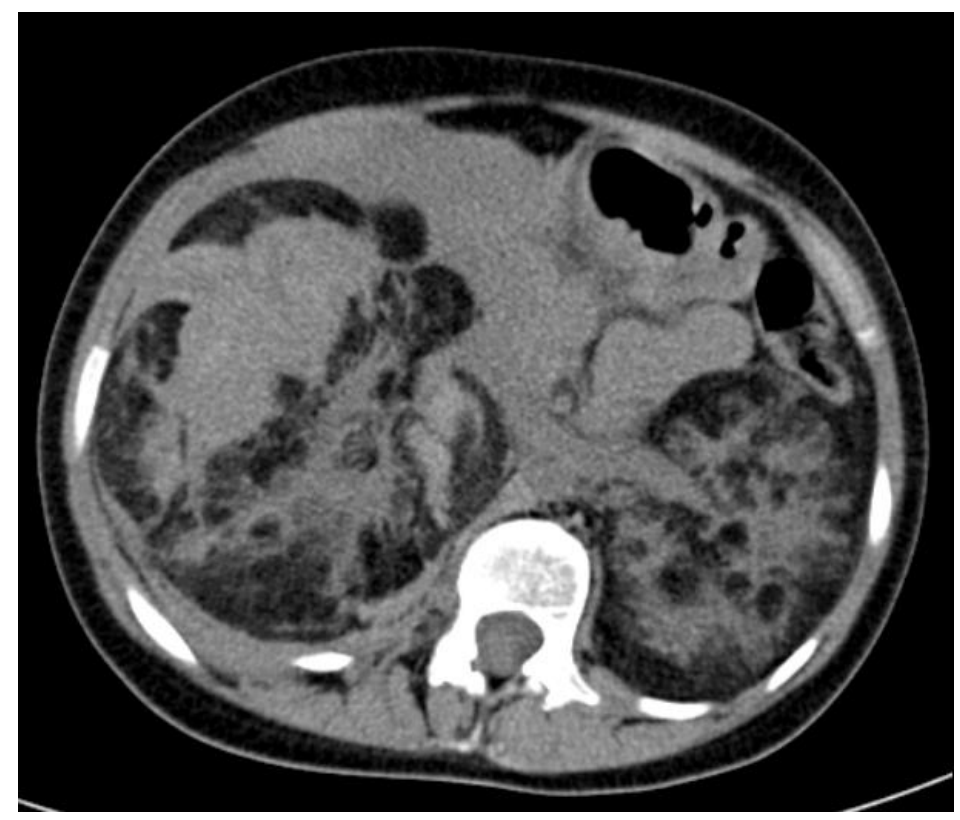

Figure 1. Unenhanced abdominal computerized tomography (sagittal scan) - instead of normal kidneys, giant cystic tumor masses are presented (with densities of fat, vessel, and muscle), with a giant right-sided retroperitoneal hematoma and intraperitoneal fluid collection.

The woman was transferred from emergency department to the operating room of the Department of Special Surgery and median laparotomy was performed immediately.
Hemoperitoneum (in amount of $400 \mathrm{ml}$ ) and a giant right-sided retroperitoneal hematoma (extending from the diaphragm to the pelvic space) were established (Figure2).

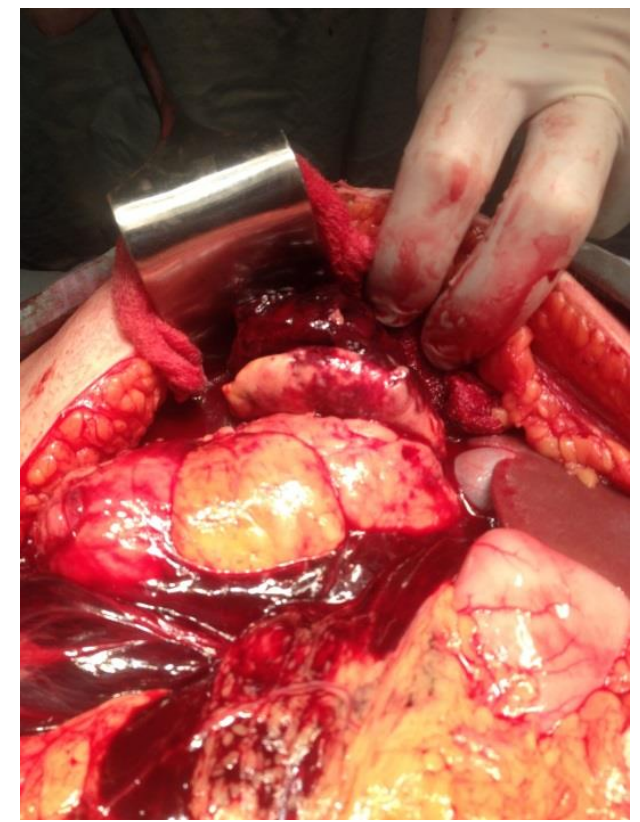

Figure 2. Intraoperative finding: a giant retroperitoneal hematoma and a giant right-sided ruptured tumor mass with active bleeding. 
It was established that hemoperitoneum was a result of blood transfer thought the disruption of the retroperitoneal parietal peritoneum. The right posterior parietal peritoneum was transected and the right retroperitoneal space was exploring. Retroperitoneal hematoma and a giant ruptured tumor with active bleeding (instead of normal right kidney) were established. The tumor was removed and the retroperitoneal area was washed. Two tube drainages were placed (in the right coloparietal region and the lesser pelvis) and the abdominal wall was closed.

Gross pathologic examination determined the removed tumor mass as "angiomyolipoma". It had irregular shape with maximum diameter of $23 \mathrm{~cm}$, with grayish - yellow colour. The transected surface of the tumor was granular (like fat tissue) with dilated thick-walled blood vessels (aneurisms) and parenchymal hemorrhage (Figure 3).

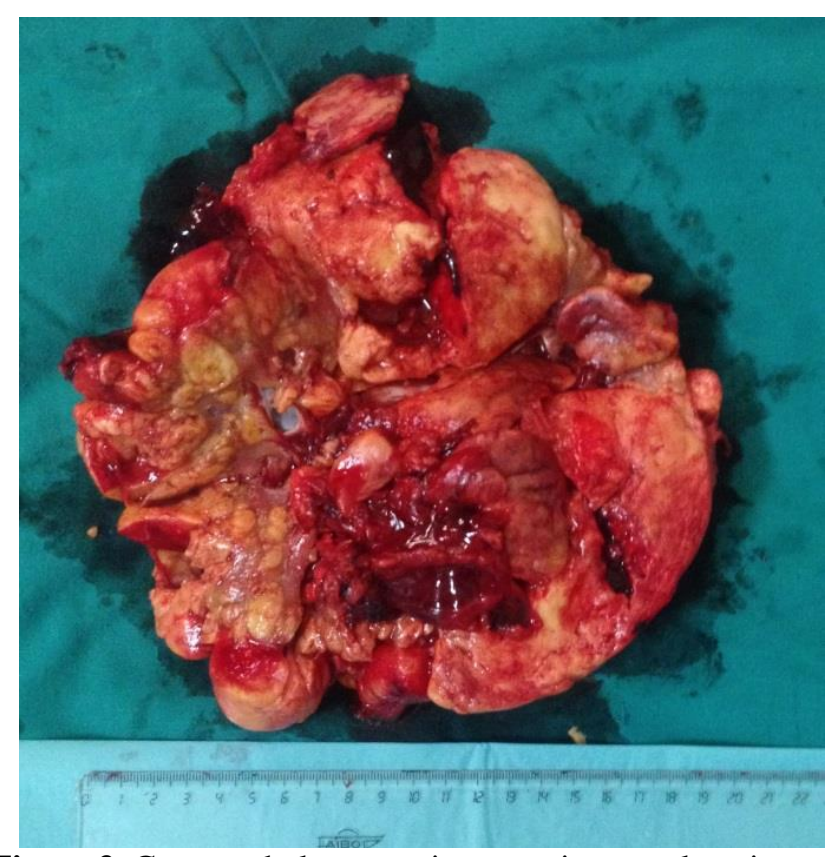

Figure 3. Gross pathology specimen: a giant renal angiomyolipoma (max. diameter of $23 \mathrm{~cm}$ ) with dilated thick-walled blood vessels (aneurisms).

The histological examination confirmed the diagnosis "angiomyolipoma": with mature adipose cells, smooth muscle, numerous thick- walled blood vessels and areas of focal hemorrhages (Figure 4).

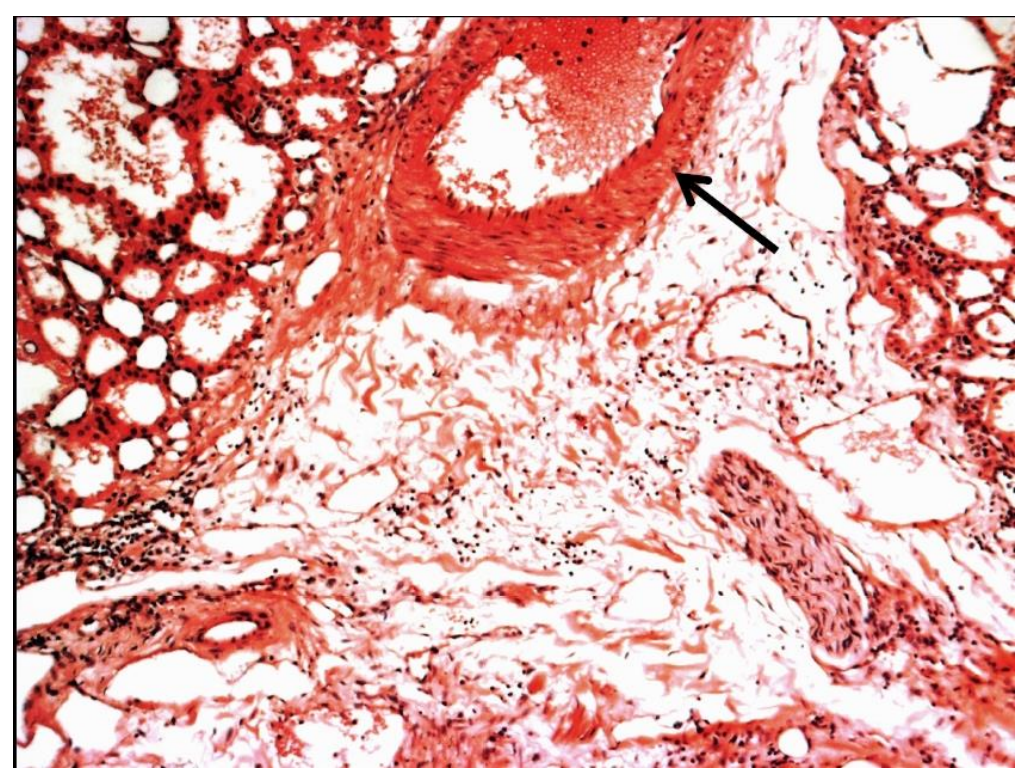

Figure 4. Photomicrograph (hematoxylin and eosin staining, $25 \mathrm{x}$ objective lens): adipose cells, smooth muscle cells, focal hemorrhages and numerous thick-walled blood vessels (aneurism is pointed with black arrow) are demonstrated. 


\section{DISCUSSION}

Angiomyolipomas of kidneys are infrequent tumors that represent $3 \%$ of solid renal masses $(1,3)$. There are two different appearances: the solitary angiomyolipoma without any sign of other disease and angiomyolipoma in cases with tuberous sclerosis complex (4-6). Solitary renal angiomyolipomas are often found incidentally when kidneys are scanned for some other reasons, with a strong prevalence of women (female:male $=4: 1$ ). Clinically, angiomyolipomas are usually silent. However, they can in rare cases cause massive retroperitoneal hemorrhage. Tuberous sclerosis complex (TSC) is an autosomal dominant disease due to a disorder of cellular migration, proliferation and differentiation (79). The incidence of tuberous sclerosis is estimated between 1:6,000 and 1:10,000 individuals and is typically characterized by the " Vogt's triad" of epilepsy, mental retardation, and facial angiofibromas (adenoma sebaceum). In more than $80 \%$ of patients with tuberous sclerosis complex there are manifestations of renal angiomyolipomas, which are multiple and bilateral. The features in the presented case were similar with those reported in the literature (8-12).

Renal angiomyolipomas arise from the mesenchymal elements of the kidney and are composed of variable amounts of mature adipose tissue, smooth muscle, thick-walled blood vessels and supporting stroma of connective tissue. There isn't integrated control of growth of these components and as a result angiomyolipoma invades adjacent normal renal parenchyma leading to chronic kidney disease and even end-stage renal disease (1-4). The most severe symptoms are associated with rupture of the tumor. This is a relatively rare complication of renal angiomyolipomas (not more that $10 \%$ of the cases with spontaneous tumor rupture)/ $(6,10-$ 12).

Of particular interest is the vascular component of renal angiomyolipomas. Angiomyolipoma's vessels are poor of elastin that makes these vascular structures prone to aneurysm formation that can rupture $(3,4,11)$.

Although most angiomyolipomas are benign and asymptomatic, symptoms develop in 68\%$80 \%$ of patients when tumor size reaches $4 \mathrm{~cm}$ or greater. There is a significant relationship between tumor size, aneurysm formation, and a risk of spontaneous rupture within renal angiomyolipomas. It had been established that the risk of this complication is very high in renal angiomyolipomas that are larger than 8 $\mathrm{cm}$ in diameter with aneurysms in their structure larger than $5 \mathrm{~mm}$, like in the presented case $(4,5,10,11,13)$.

The spontaneous rupture can cause intrarenal (parapelvic) bleeding, subcapsular hematomas or bleeding in the posterior perirenal space. Some cases with spontaneous rupture of renal angiomyolipomas are presented with symptoms of hypovolemic shock - lifethreatening condition knowing as "Wunderlich's syndrome" $(11,13,14,15)$.

High index of suspicion of ruptured renal angiomyolipoma with retroperitoneal bleeding rises from patient's complaints and physical examination (5). Imaging methods like unenhanced abdominal computed tomography must be done for rapid confirmation of this severe complication, just like in the presented case.

The management of ruptured renal angiomyolipoma is dictated by the severity of the bleeding and clinical condition of the patient. It may vary from conservative treatment (fluid resuscitation), through selective arterial embolization to the surgery (partial or total nephrectomy). In Wunderlich's syndrome with retroperitoneal hemorrhage partial or total nephrectomy is the treatment option in order to safe the patient's life $(11,15$, 16). The operation may be performed through retroperitoneal or abdominal approach. Abdominal approach can be done by laparoscopic or open (conventional) surgery by laparotomy. There are reports of minimal invasive procedures $(3,4,7,11,13)$. However, we consider urgent median laparotomy as the method of choice in the management in massive retroperitoneal bleeding as a result of spontaneous rupture of renal angiomyolipoma with imagines data of intraperitoneal blood collection. Median laparotomy ensures exploration of the peritoneal cavity and retroperitoneal space, removing the bleeding renal tumor as well as adequate peritoneal and retroperitoneal lavage and drainage.

\section{CONCLUSION}

In conclusion, this case presents rare, but the most severe complication of renal angiomyolipomas in a relatively young patient with tuberous sclerosis complex - spontaneous rupture of the tumor with life-threatening 
retroperitoneal bleeding. The presenting case confirmed the conventional approach - median laparotomy as method of choice in the management of such patients with massive retroperitoneal bleeding due to the spontaneous rupture of giant renal angiomyolipoma.

\section{REFERENCES}

1. Dixon BP, Hulbert JC, Bissler JJ. Tuberous sclerosis complex renal disease. Nephron Exp Nephrol, 118:15-20, 2011.

2. Azevedo AS, Nábia MM, Simão S. Multicentric angiomyolipoma in kidney, liver, and lymph node: case report/review of the literature. J Bras Pathol Med Lab, 51:173-177, 2015.

3. Eble JN. Angiomyolipoma of kidney. Seminars Diag Pathol, 15:21-40, 1998.

4. Moratalla MB. Wunderlich's syndrome due to spontaneous rupture of large bilateral angiomyolipomas. Emergency Medicine Journal, 26:72, 2009

5. Cui LJ, Zhang G, Hu XJ. CT imaging and histopathological features of renal epithelioid angiomyolipomas. Clinical Radiology, 67:77-82, 2012.

6. Chen YC, Lin YC. Wunderlich syndrome. QJM, 16:187-188, 2013.

7. Faddegon S, So A. Treatment of angiomyolipoma at a tertiary care centre: the decision between surgery and angioembolization. Journal of the Canadian Urological Association, 5:138141, 2011.

8. Harrison JE, Bolton PF. Annotation: Tuberous sclerosis. $J$ of Child Psychology and Psychiatry, 386:603-614, 1997.

9. Bradley P, Dixon JC, Hulbert JJ. Tuberous
Sclerosis Complex. Renal Disease Nephron Exp Nephrol,118:15-20, 2011.

10.Nabi N, Shaikh AH, Khalid SE. Tuberous sclerosis with bilateral renal angiomyolipoma and Wunderlich's syndrome. J Coll Physicians Surg Pak, 17:706-707, 2007.

11.Granata A, Basile A, Figuera M. et al. Spontaneous retroperitoneal hemorrhage due to massive rupture of renal angiomyolipoma treated with nephrectomy: an unusual onset of tuberous sclerosis complex. Clinical Nephrology, 71:441 444, 2009.

12.Kulkarni B, Desai SB, Dave B. Renal angiomyolipomas - a study of 18 cases. Indian J of Pathol Microbiol, 48: 459-463, 2005.

13. Chronopoulosa PN, Kaisidisa GN, Vaiopoulosa CK. Spontaneous rupture of a giant renal angiomyolipoma Wunderlich's syndrome: report of a case. Intern J Surg Case Reports, 19:140-143, 2016.

14. Mouded IM, Tolia BM, Bernie JE, Newman HR. Symptomatic renal angiomyolipoma: report of 8 cases, 2 with spontaneous rupture. J Urol, 19:684-688, 1978.

15.Lewis EL, Palmer JM. Renal angiomyolipoma and massive retroperitoneal hemorrhage during pregnancy. West J Med, 143:675-6, 1985.

16. Ortega-Deballon P1, Ruiz-Rubio JL, Sirvent-Cerdá S, Moreno-Azcoita M. Spontaneous hemorrhage from renal angiomyolipoma. Am J Surg, 180:54, 2000. 